\title{
Simulación de tráfico en circuitos urbanos para analizar el efecto de diferentes estilos de conducción
}

\author{
Felipe Jiménez Alonso \\ INSIA - Universidad Politécnica de Madrid \\ Carlos Dómine Rueda \\ Universidad Politécnica de Madrid
}

\section{RESUMEN}

El artículo presenta un simulador de flujo de tráfico en entornos urbanos orientado a analizar el efecto sobre cada vehículo y sobre el conjunto del tráfico de diferentes estilos de conducción. El simulador permite la configuración de los parámetros de circulación de vehículos "controlados", así como del resto de vehículos con los que pueden tener interrelación. La configuración abierta del entorno de simulación permite la carga sencilla de cualquier circuito que vaya a realizar el vehículo "controlado", incluyendo los elementos de la infraestructura más influyentes, así como el patrón objetivo de circulación de dicho vehículo que caracteriza el estilo de conducción a estudiar, y se configura el resto del tráfico a partir de parámetros que pueden ser fácilmente medidos o estimados.

Esta aplicación permite estimar, entre otras variables, consumos o tiempos de viaje en diferentes escenarios de intensidad de tráfico, así como ante diferentes patrones de conducción. También se permite la introducción de variables del vehículo. Por otra parte, es posible analizar la interrelación entre el vehículo "controlado" y el tráfico, estudiando cómo influye cada uno en la evolución del otro.

Frente a modelos más complejos de simulación, tiene la ventaja de que resulta muy intuitivo, los parámetros de tráfico necesarios son simples de medir y los resultados obtenidos son fácilmente interpretables, integrándose tanto variables macroscópicas de tráfico como microscópicas a nivel de cada vehículo, pudiendo modelizar con detalle cada tipología de conductor, pudiendo seguir la evolución de cualquiera de ellos.

\section{INTRODUCCIÓN}

A la hora de analizar el movimiento y trayectoria de un vehículo, tenemos que tener en cuenta que estas variables están condicionadas por una serie de factores controlables por el conductor, como son las aceleraciones, deceleraciones y cambios de velocidad voluntarios en general, y por otros que responden a la naturaleza del circuito, como puedan ser las velocidades máximas en cada tramo de un circuito; el movimiento de otros vehículos que, yendo delante del analizado, condicionan su movimiento; los semáforos, y otras señales de 
tráfico.

Esta relación entre los distintos vehículos puede ser modelizada desde diferentes perspectivas, distinguiéndose los modelos macroscópicos (Greenberg, 1959; Edie, 1961; Esser y Schreckenberg, 1997) y los microscópicos (Au, Zhang, Stone, 2015). Cada uno de ellos plantea ventajas e inconvenientes. En el modelo que se presenta en este artículo se ha pretendido un análisis microscópico donde se observen las interacciones entre un vehículo y el resto, y la repercusión global sobre el tráfico.

El modelo planteado se enfoca desde un estado simple de modelo de tráfico sobre circuito cerrado de un conjunto de vehículos que pueden entrar o salir de él, poniendo el foco del análisis sobre un vehículo concreto individual que se mueve en dicho entorno. Esta arquitectura permite la incorporación natura de dos desarrollos de mayor complejidad:

- Modelización de las intersecciones, principalmente de las no reguladas por semáforos según los resultados ofrecidos por modelos complejos (Brilon, 1991; Troutbeck, 1986)

- Modelización realista del comportamiento de cada conductor, en base a clasificaciones realizadas con herramientas de Inteligencia Artificial para configurar el tráfico real

\section{MODELO DE TRÁFICO BÁSICO}

El modelo de tráfico básico simula el movimiento de una serie de vehículos situados a lo largo del circuito cerrado en función del tiempo (Figura 1). En cada segundo, se simula la posición de todos los vehículos. Básicamente se definen dos tipos de vehículos: vehículo principal o "controlado", del cual podremos controlar su velocidad en todo momento y aquellos vehículos que completan el tráfico en el circuito y que tendrán características homogéneas en un primer estado de desarrollo. Mientras que estos vehículos se mueven en función de las velocidades medias tomadas como datos, el vehículo principal podrá circular de la forma que se desee, simulando diferentes estilos de conducción y cómo el tráfico circundante influye sobre él y viceversa. De esta forma se simula un flujo de vehículos en cuya trayectoria no podemos influir, y uno totalmente autónomo que podremos controlar. En el modelo se obvia, por lo tanto, el hecho de que un vehículo pueda adelantar a otro en ciertas zonas, pero realmente es algo que no influye, ya que todos los vehículos simulados son iguales e indistinguibles entre sí, salvo el coche principal o autónomo, el cual podría adelantar únicamente en las zonas en las que estuviese permitido o hubiese más de 1 carril. 


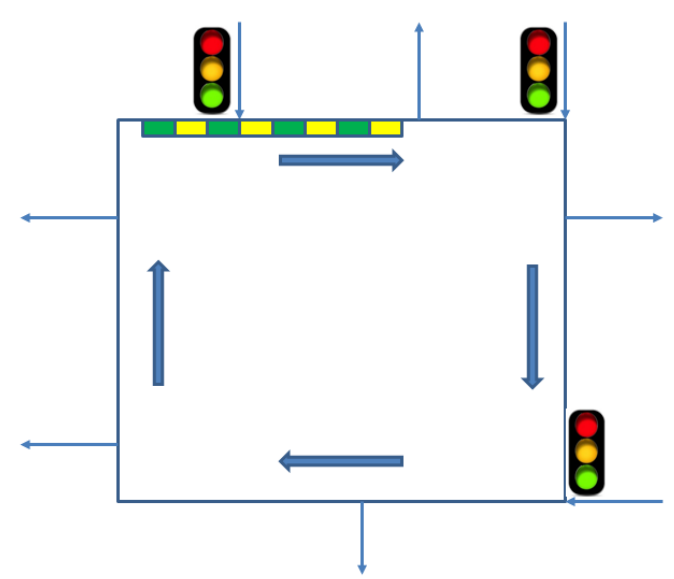

Fig. 1 - Esquema del modelo básico de tráfico

Antes de evaluar el movimiento de los vehículos en cada ciclo, el modelo controla 2 aspectos:

- Ciclos verde - rojo de los semáforos en cada paso de ejecución del programa

- Vehículos que deben entrar o salir en cada intersección (regulada o no por semáforos) en cada ciclo de ejecución

Al comienzo de la simulación el circuito debe tener una serie de vehículos dispuestos de forma aleatoria a lo largo del trayecto. Por otra parte, en las intersecciones, el modelo trabaja sobre las probabilidades de entrada o salida en ellas que se miden experimentalmente. Sin embargo, la introducción o salida de vehículos está condicionada por el hecho de que las celdas de la intersección estén ocupadas o vacías (si está vacía no podría salir un vehículo y si está ocupada no podría introducirse un vehículo). Para que las entradas y salidas efectivas sean realistas, se multiplican los valores experimentales por los denominados coeficientes de intersecciones. Estos coeficientes se calculan de la siguiente manera:

$$
\begin{aligned}
& C E=\frac{C_{T}}{C_{T}-C_{O}+1} \\
& C S=\frac{C_{T}}{C_{O}+1}
\end{aligned}
$$

donde

- CE: coeficiente de vehículos entrantes

- CS: coeficiente de vehículos salientes

- $\quad \mathrm{C}_{\mathrm{T}}$ : número total de celdas

- Co: número de celdas ocupadas (vehículos) inicialmente.

A continuación, se simula la posición en cada segundo de todos los vehículos. Para ello, el circuito se divide en secciones individuales, las cuales pueden encontrase en 3 estados: vacía, ocupada por el vehículo principal u ocupada por un vehículo del tráfico. Los vehículos se 
mueven siguiendo pautas según las secciones del recorrido donde se marcan velocidades medias o por la interacción con las intersecciones. De igual forma, el vehículo controlado sigue un patrón preestablecido de velocidades en el circuito, salvo interacciones con otros vehículos. En general, las velocidades se cuantifican en celdas/s, y no están limitados, aunque este aspecto no supone una limitación excesiva ya que la resolución de velocidades se puede aumentar reduciendo el tamaño de las celdas. Para el estudio del vehículo controlado, los semáforos y el resto de vehículos del circuito serán, por lo tanto, los elementos simulados que impedirán a este vehículo ir a la velocidad deseada, retrasando su recorrido.

La simulación ofrece las variables principales del vehículo "controlado", ofreciendo las siguientes salidas:

- velocidad media incluyendo paradas;

- velocidad media sin incluir paradas;

- número de vueltas completas dadas al circuito;

- distancia total recorrida;

- tiempo parado;

- número de paradas.

- número de aceleraciones;

- número de deceleraciones;

\section{EJEMPLO DE SIMULACIÓN}

A continuación se muestran a modo de ejemplo, algunos resultados sobre una ruta circular en los alrededores de Madrid (Figura 2)

\section{Fig. 2 - Ruta simulada}

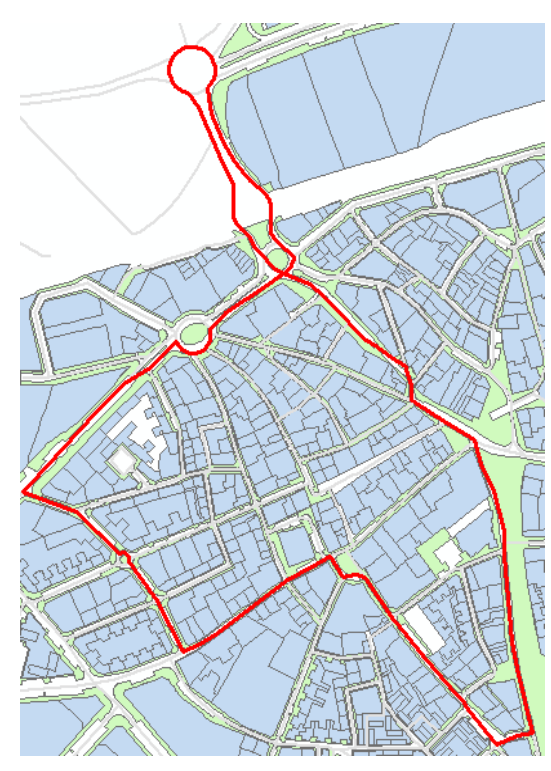

El recorrido tiene una longitud de unos $2,41 \mathrm{~km}$, sobre los cuales se requieren las medidas 
experimentales siguientes:

- semáforos, de los cuales nos interesan los ciclos de estancia en verde y rojo y los vehículos acumulados durante este último ciclo;

- intersecciones con otras carreteras ajenas al circuito, en los cuales se toman datos de los vehículos que entran y los que salen.

- velocidades medias de los vehículos en cada tramo del circuito, fundamentales para poder simular de forma veraz el movimiento y los tiempos de los vehículos.

El ejemplo simulado considera tasas de entradas y salidas totales nulas con lo que, en un horizonte temporal largo, el número de vehículos se debe mantener constante en el valor inicial. Sin embargo, la aleatoriedad de los parámetros de entradas y salidas propicia que se presenten oscilaciones como muestra la Figura 3.

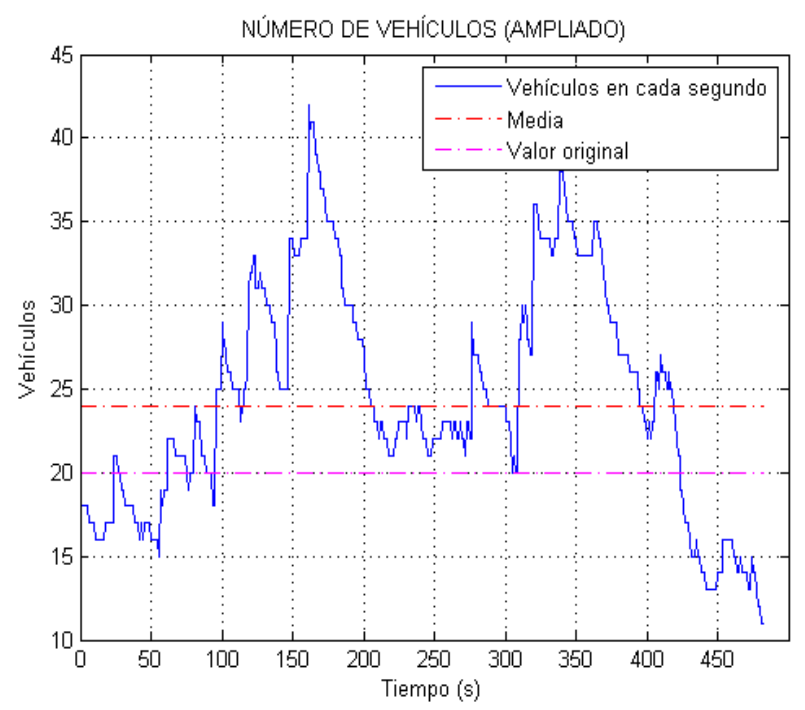

\section{Fig. 3 - Ejemplo de evolución del número de vehículos del circuito}

En la Figura 4 se observa la evolución de los vehículos a lo largo de las celdas del circuito. Podemos ver como hay celdas en las cuales desaparecen algunos vehículos, y otras donde aparecen otros nuevos: son los cruces. De igual forma se pueden visualizar perfectamente los atascos, como grandes cúmulos de puntos. De igual forma, los cambios de velocidad en la trayectoria de un vehículo se ven reflejados en cambios en las pendientes de las líneas. 


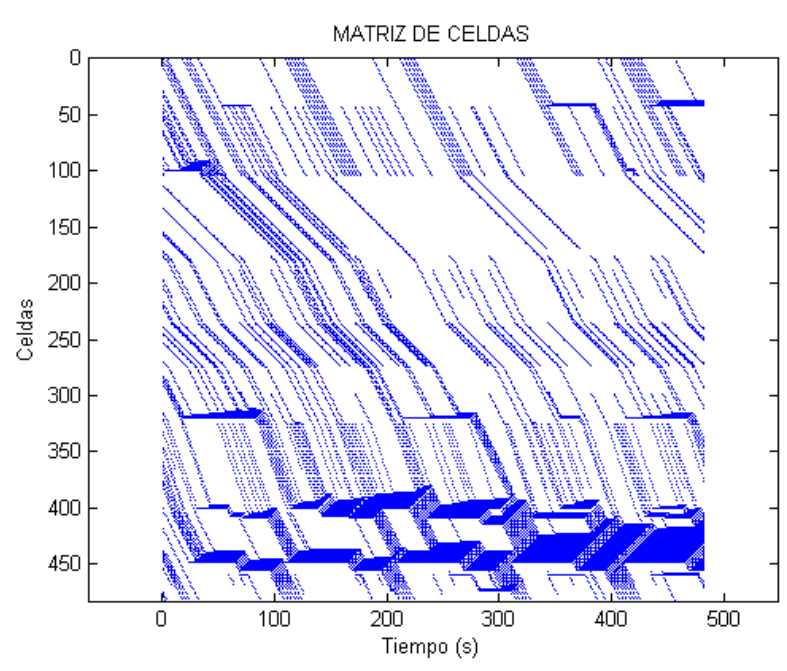

Fig. 4 - Evolución de los vehículos en el circuito

Se puede apreciar cómo se van formando y diluyendo los atascos, mediante ondas de choque, en las cuales tanto las frenadas de los vehículos como sus puestas en marcha se van transmitiendo de uno a otro. También hay que señalar que, mientras que en los atascos los vehículos pueden llegar a él de forma independiente, sin ir en grandes grupos, como al salir de él todos los vehículos irán en un mismo grupo, uno detrás de otro. Además en esta figura se ve reflejada una situación muy común en este circuito, que son los semáforos cercanos, de tal forma que en el centro de la imagen podemos ver dos grandes atascos y otro más pequeño, cada uno creado por un semáforo distinto. Si los ciclos en rojo fuesen más altos, esos tres atascos habrían acabado fusionándose en uno solo.

La tabla 1 muestra los resultados de las variables del vehículo controlado ante dos situaciones operativas diferentes.

\begin{tabular}{|l|c|c|}
\cline { 2 - 3 } \multicolumn{1}{c|}{} & $\begin{array}{c}\text { Conducción normal } \\
\text { y tráfico medio }\end{array}$ & $\begin{array}{c}\text { Conducción agresiva } \\
\text { y tráfico alto }\end{array}$ \\
\hline $\begin{array}{l}\text { Velocidad objetivo del vehículo } \\
\text { controlado }(\mathrm{km} / \mathrm{h})\end{array}$ & Promedio del tráfico & 54 \\
\hline Vehículos iniciales & 70 & 100 \\
\hline velocidad media con paradas $(\mathrm{km} / \mathrm{h})$ & 13.3 & 11.4 \\
\hline velocidad media sin paradas $(\mathrm{km} / \mathrm{h})$ & 29.4 & 29.9 \\
\hline número de vueltas & 13 & 11 \\
\hline distancia total recorrida $(\mathrm{k})$ & 33.3 & 28.6 \\
\hline tiempo parado $($ min) & 82. & 92.8 \\
\hline número de aceleraciones & 281 & 337 \\
\hline número de deceleraciones & 322 & 398 \\
\hline número de paradas & 227 & 282 \\
\hline
\end{tabular}

Tabla 1 - Comparación de resultados del vehículo controlado 
En los resultados se muestra claramente cómo incluye el resto del tráfico en el vehículo controlado, aún a pesar de su consigna inicial. Esto se debe a que la ocupación del circuito es bastante altas (cercana a un tercio del total). Así, se comprueba mediante la simulación del efecto entre el tráfico y la circulación de un vehículo concreto.

La Figura 5 recoge la influencia de la velocidad de seguimiento (velocidad que el vehículo controlado tiende a alcanzar en los distintos tramos del recorrido).manteniendo el número inicial de vehículos en 70 . Se comprueba que fijar una velocidad objetivo para el vehículo controlado de 4 celdas $/ \mathrm{s}(72 \mathrm{~km} / \mathrm{h}$ ) dará prácticamente el mismo resultado que si la velocidad máxima fuese de 2 celdas $/ \mathrm{s}$ ( $36 \mathrm{~km} / \mathrm{h}$ ). El resto de elementos del circuito limitarán de tal manera la trayectoria del vehículo autónomo que prácticamente no le dejan espacios en el recorrido para circular a la velocidad deseada; esta estará la mayor parte del tiempo limitada.

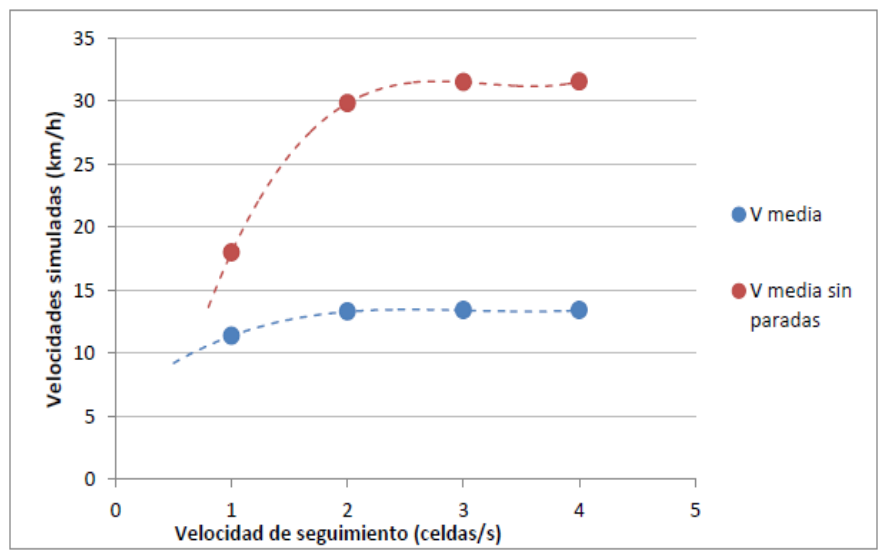

Fig. 5 - Influencia de la velocidad de seguimiento

La Figura 6 muestra la influencia de la densidad de tráfico donde se supone un conductor controlado que quiere circular siempre a 3 celdas $/ \mathrm{s}(54 \mathrm{~km} / \mathrm{h})$. Variando el número de vehículos iniciales se aprecia que la influencia es mayor con densidades bajas y que, incrementos en esta variable a mayores densidades provoca un efecto relativo inferior sobre la velocidad de circulación.

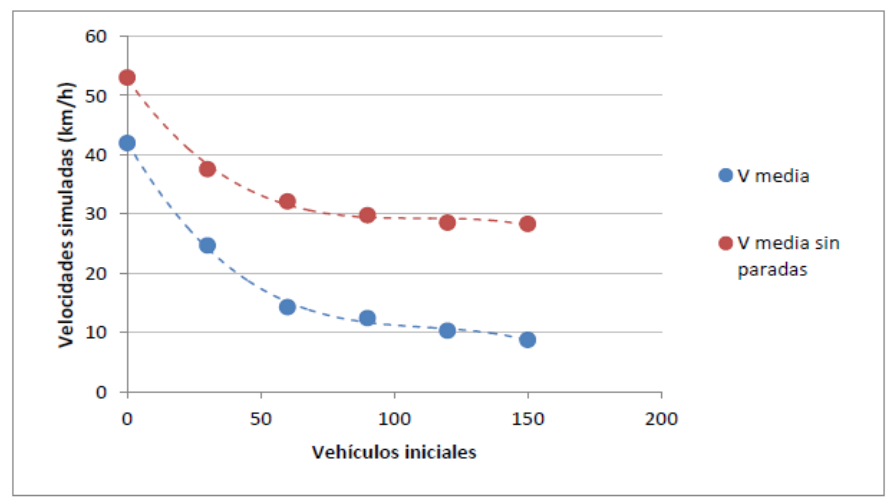

Fig. 6 - Influencia de la densidad de tráfico 
Por último, la Figura 7 comprende ambos efectos simultáneamente. Comparando las tres curvas vemos que la verde adquiere una forma casi horizontal con un valor cercano a 10 $\mathrm{km} / \mathrm{h}$. Con una densidad de tráfico de 120 vehículos iniciales el estilo de conducción que lleve un conductor controlado, normal o agresivo, carece de relevancia a la hora de obtener resultados, pudiendo aumentar una conducción agresiva la velocidad final en valores del orden de $1 \mathrm{~km} / \mathrm{h}$. En la curva roja, con 70 vehículos iniciales, sí se empiezan a apreciar las influencias del estilo de conducción a la hora de obtener resultados, circulando más rápido y pudiendo, por lo tanto, recorrer una mayor distancia en el mismo tiempo. En este caso las velocidades finales tienen una variación mayor según la velocidad a alcanzar sea 1, 2 o 3 celdas $/ \mathrm{s}(18,36,54 \mathrm{~km} / \mathrm{h})$, pero siguen siendo intervalos muy pequeños en comparación con el cambio que supone querer duplicar o triplicar una determinada velocidad. Solamente con densidades bajas de tráfico, como la representada en la curva azul, tendremos una relación más acusada entre la velocidad deseada por el conductor y la efectiva que obtiene tras la simulación. Sin embargo, aun en este caso vemos que la línea de tendencia hace que, a pesar de tener más libertad de circulación, si quisiéramos circular a velocidades muy altas el propio circuito limitaría dicha decisión. Concluyendo, el modelo demuestra resultados coherentes en los que, cuanto menor sea la densidad de tráfico, más influye la velocidad que se desea alcanzar sobre la velocidad final del vehículo. Sin embargo, conforme aumenta la densidad de tráfico, esta velocidad se va haciendo más irrelevante hasta tener consecuencias prácticamente nulas.

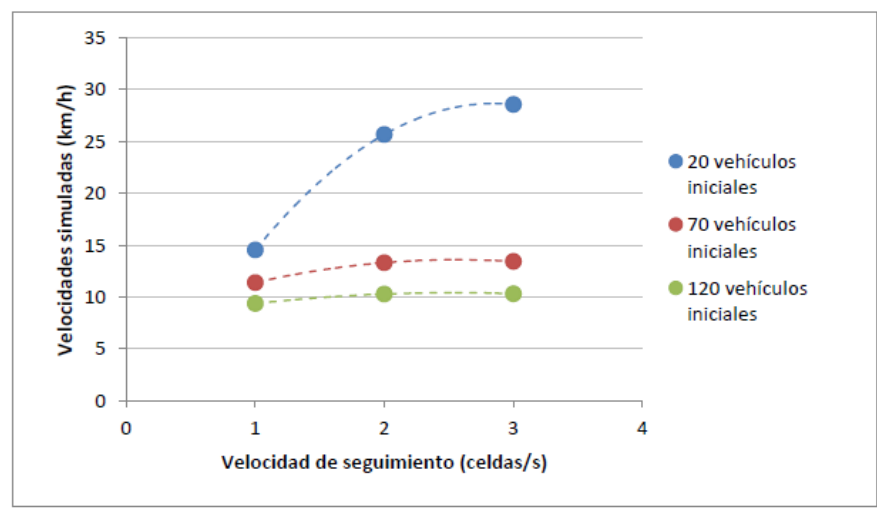

Fig. 7 - Influencia de la densidad de tráfico y la velocidad de seguimiento

\section{MODELO DE INTERSECCIONES}

El modelo anterior adolece de una simplificación excesiva en el tratamiento de las intersecciones. En concreto, se pretende dotar de un comportamiento más realista a los vehículos que entran y salen en dichas intersecciones teniendo en cuenta variables como las presentadas en modelos de Brilon (1991) y Troutbeck (1986).

De igual forma, se generaliza el modelo anterior de circuito cerrado en un circuito en malla como el representado en la figura 8 en el que cada segmento está caracterizado por las variables siguientes: 
- Número de carriles en cada sentido.

- Número máximo de vehículos que pueden estar detenidos en el segmento

- Velocidad de circulación en el segmento con flujo libre

De igual forma, en cada intersección, se indican las siguientes variables:

- Ciclos vede-rojo de las intersecciones reguladas por los semáforos

- Giros permitidos

- Porcentaje de vehículos que adoptan cada uno de los giros permitidos

Por último, la malla es alimentada por los vehículos que se introducen por los nodos periféricos con opción de entrada y elimina vehículos por los nodos periféricos con opción de salida. De os primeros se conoce la tasa de vehículos por unidad de tiempo que entran en el sistema, siendo los vehículos que salen un número libre que vendrá condicionado por la modelización de la malla. En su aplicación práctica, serán estas tasas de salida lo que permita ajustar el modelo.

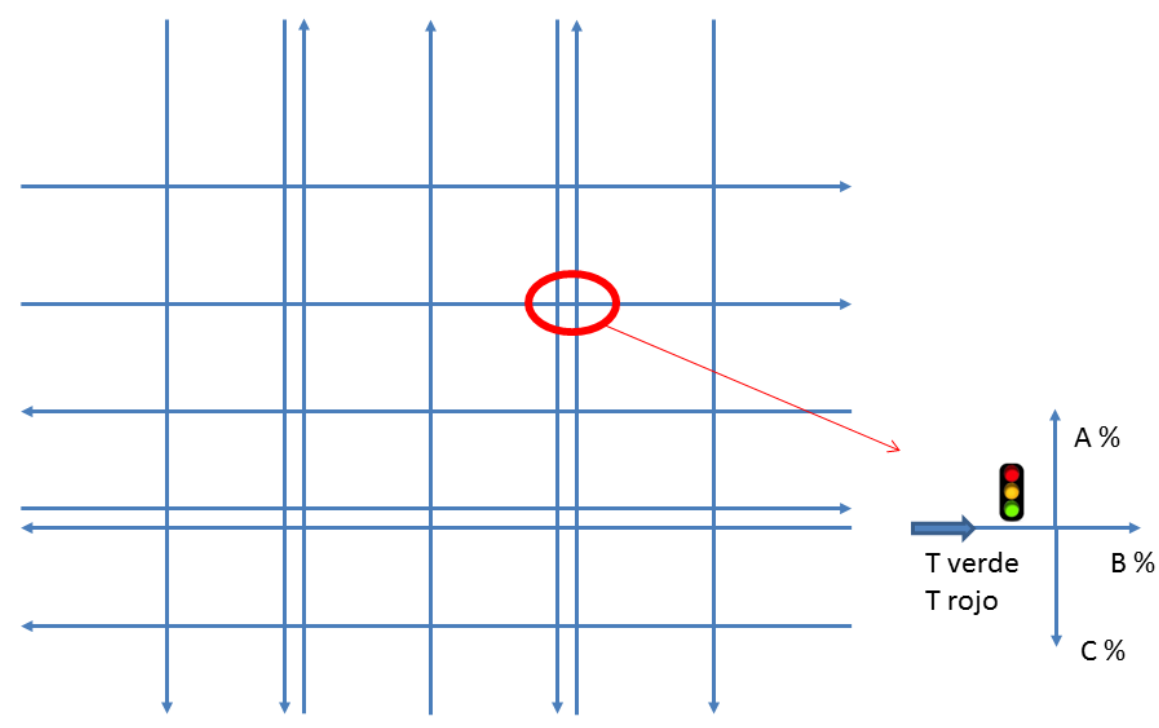

Fig. 8 - Esquema de circuito en malla

Sobre el esquema de modelo anterior, es posible analizar estrategias de gestión semafórica y, entre ellas, la implantación de medidas como "ondas verdes" para vehículos de transporte público colectivo de pasajeros (Furth, 2005)

\section{MODELO DE COMPORTAMIENTO DE CONDUCTOR}

Finalmente, la última extensión del modelo de simulación de tráfico implica la modelización en detalle del conductor. Para ello, se recurre a la aplicación de técnicas de la rama subsimbólica de la Inteligencia Artificial sobre el área de la conducción, para el análisis del comportamiento de conductor (detección de los modelos y patrones de eficiencia y agresividad que subyacen en los comportamientos de los conductores). Debe indicarse que 
ya se han explorado estas técnicas en este ámbito pero con una orientación hacia la conducción eficiente (Díaz et al, 2014).

Es decir, se pretende realizar un modelo del perfil de conducción a partir de técnicas de Inteligencia Artificial y aplicándolo en un entorno multiagente de donde obtener el resto de parámetros. Así, una vez configurado el entorno multiagente, se podrá simular el tráfico focalizando el análisis en la forma de conducir de cada uno cuando su marcha está condicionada por el tráfico, los semáforos, etc.

Debe tenerse en cuenta que, en este ámbito y de forma general, identificar a un conductor no es fácil, dado que su comportamiento se ve condicionado por el estado de la ruta y su tráfico. Comportamientos considerados en algunos casos como agresivos pueden deberse a situaciones límite condicionadas por las malas reacciones por parte de otros conductores. Además, la identificación de conductas eficientes muchas veces subyace al observarse el comportamiento de conductor ante el entorno (otros vehículos, ciclos e semáforos, etc).

Analizar todos los posibles casos en los que un mismo conductor puede encontrarse es un problema muy costoso, cuanto más disponer de un conjunto lo suficientemente amplio de sujetos de estudio para analizar cada uno de dichos casos. Por ello, las simulaciones pueden dar una estimación de los posibles resultados de un estudio en el mundo real.

Los sistemas multiagente (SMA o MAS del inglés Multi-Agent Systems) son aquellos sistemas compuestos por diversos elementos denominados agentes, los cuales cooperan sobre un entorno para, normalmente, llegar a una solución. Muchos de estos sistemas se usan en simulaciones donde cada uno de los agentes modela un individuo o problema para, a través de iteraciones discretas de tiempo, evaluar el comportamiento en general del sistema en base a sus individuos.

Las simulaciones con sistemas multiagente donde se insertasen agentes obtenidos de la modelización de conductores a partir de sus datos en experimentos de campo podrían ser consideradas como fuente de datos para aquellas condiciones de tráfico y/o ruta no contempladas en dichas pruebas. De esta forma, se dispondría de un marco de trabajo para la comparación de diferentes conductores sin necesidad de exponerlos a todos y cada uno de los posibles eventos posibles. También sería posible evaluar sistemas de asistencia evitando los problemas de no comparabilidad de condiciones del entorno entre pruebas.

Dentro de este marco, se están explorando dos opciones de introducción de datos a los agentes que reproducen los conductores:

- Identificación de los parámetros del agente (parámetros de conducción) y origen - destino de cada uno, operación que se repetiría con todos los usuarios que se introducirían en el circuito 
- Flujos de entrada como en el caso del modelo en malla, pero incluyendo la información de cada agente para que cada vehículo sea conducido de una forma particular

La primera opción presenta una clara ventaja en cuanto al detalle con el que se puede incidir y por el hecho de que corresponde al estudio individualizado de cada usuario (el cual decide origen, destino y puede ser descrito por unas variables de conducción). Esta opción, en un entorno real de una ciudad sería altamente tediosa, pero acepta formas sistemáticas de introducción de vehículos. La segunda opción presenta la gran ventaja de que no requiere conocer las matrices origen-destino para cada vehículo (lo que es complicado de establecer, en general) y basta con conocer flujos entrantes, más fáciles de medir, y bastaría con validar los modelos con los flujos salientes, por ejemplo.

\section{CONCLUSIONES}

Se ha conseguido desarrollar un programa en MATLAB capaz de simular con gran versatilidad circuitos urbanos amoldándose a sus distintas características. El programa trabaja con grandes cantidades de datos, si bien los parámetros de entrada básicos para ofrecer simulaciones rápidas son sencillos de obntener de forma experimental sobre un circuito, lo que hace que el programa tenga claras aplicaciones docentes.

Por otra parte, el modelo básico se ha evolucionado para integrar un sistema en malla de calles que reproduce más fielmente un entorno urbano de forma que se perfecciona el comportamiento en las intersecciones.

Por último, los últimos desarrollos que se están llevando a cabo van dirigidos hacia una modelización más fina del comportamiento de cada conductor según parámetros modelizables empleando técnicas de Inteligencia Artificial, superando algunas limitaciones de los enfoques más deterministas o estadísticos.

\section{AGRADECIMIENTOS}

Este trabajo ha sido parcialmente financiado por el Ministerio de Economía y Competitividad (proyecto TRA2013-48314-C3-2-R) y la Red de Excelencia de la Comunica de Madrid SEGVAUTO-TRIES. Los autores también quieren agradecer las aportaciones de Álvaro Antona en la programación del modelo de intersecciones.

\section{REFERENCIAS}

AU, T.-C., ZHANG, S., STONE, P. (2015). Autonomous Intersection Management for Semi-Autonomous Vehicles. En Handbook of Transportation, Routledge, Taylor \& Francis Group 
BRILON, W. (1991). Intersections without traffic signals. Berlin : Springer Publications

EDIE, L.C. (1961). Car-Following and Steady-State Theory for Noncongested Traffic. Operations Research 9(1), p 66-76.

DÍAZ, A., SERRADILlA, F., NARANJO, J. E., ANAYA, J. J., JIMÉNEZ, F. (2014). Modeling the Driving Behavior of Electric Vehicles Using Smartphones and Neural Networks. IEEE Intelligent Transportation Systems Magazine. vol 6, n 3, pp 44-53

ESSER, J., SCHRECKENBERG, M. (1997) Macroscopic Simulation of Urban Taffic Based on Cellular Automata. International Journal of Modern Physics C, 8(5), pp. 10251036

FURTH, P. G. (2005). Public Transport Priority for Brussels: Lessons from Zurich, Eindhoven, and Dublin. Report Completed Under Sponsorship of the Brussels Capital Region Program "Research in Brussels"

GREENBERG, H. (1959) An Analysis of Traffic Flow. Operational Research, 7 (1), pp 7985

TROUTBECK, R. J. (1986). Average delay at an unsignalized intersection with two major streams each having a dichotomized headway distribution. Transportation Science, 20 (4), pp 272-286 